Diabetologe 2019 $\cdot 15: 6$

https://doi.org/10.1007/s11428-018-0427-7

(c) Springer Medizin Verlag GmbH, ein Teil von Springer Nature 2018

CrossMark

Klaus G. Parhofer

Medizinische Klinik IV - Großhadern, Klinikum der Universität München, München, Deutschland

\title{
Lipidstoffwechselstörungen und deren Behandlung bei Diabetes mellitus
}

Evidenz belegter medikamentöser Ansätze verfügbar (Statine, Ezetimib, PCSK9Inhibitoren [PCSK9: Proproteinkonvertase Subtilisin/Kexin Typ 9]).

\section{》) Fettstoffwechselstörungen müssen bei Diabetes adäquat behandelt werden}

Allerdings haben Patienten mit Diabetes mellitus und Hypertriglyzeridämie selbst dann ein erhöhtes Risiko, wenn die LDL-Cholesterin-Zielwerte $(<100 \mathrm{mg} / \mathrm{dl}$; $<70 \mathrm{mg} / \mathrm{dl}$ ) erreicht werden. Ein weiterer Fokus ist deshalb die Absenkung erhöhter Triglyzeridspiegel und damit das Erreichen des Non-HDL-Zielwerts. Hierbei spielen Lebensstilmaßnahmen und Blutzuckerspiegeleinstellung eine überragende Rolle. Allerdings gibt es inzwischen auch eine neuere Studie mit Omega-3-Fettsäuren [1], in welcher ein signifikanter Nutzen hinsichtlich der Reduktion kardiovaskulärer Ereignisse beschrieben wurde.

Auch aus physiologischer bzw. pathophysiologischer Sicht ist der Zusammenhang zwischen Lipid- und Glukosestoffwechsel und damit zwischen Diabetes mellitus und Lipidstoffwechselstörungen hochinteressant. So haben Patienten mit Diabetes mellitus nicht nur oft einen veränderten Lipidstoffwechsel, sondern Veränderungen desselben können auch den Glukosestoffwechsel beeinflussen. Daneben ist bekannt, dass Antidiabetika den Lipidstoffwechsel beeinflussen können (nicht nur indirekt über eine bessere Blutzuckerspiegeleinstellung, sondern auch direkt) und genauso Lipidsenker die Blutzuckerspiegeleinstellung. Von besonderem Interesse ist hierbei die Beeinflussung des Glukosestoffwechsels durch Statine.

Zusammenfassend führte die stärkere Berücksichtigung des Lipidstoffwechsels bei der Behandlung von Patienten mit Diabetes mellitus zu einer deutlichen Prognosebesserung. Eine adäquate Behandlung von Fettstoffwechselstörungen ist deshalb ein zentrales Anliegen des Diabetesmanagements.

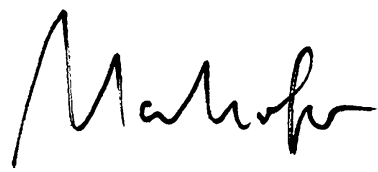

Prof. Dr. Klaus G. Parhofer

\section{Korrespondenzadresse

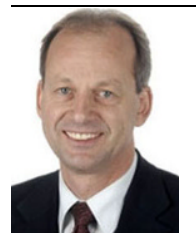 \\ Prof. Dr. Klaus G. Parhofer Medizinische Klinik IV - Großhadern, Klinikum der Universität München Marchioninistr. 15, 81377 München, Deutschland Klaus.Parhofer@med.uni- muenchen.de}

Interessenkonflikt. K.G. Parhofer erhielt Vortragshonorare, Honorare für Advisory-Board-Tätigkeit, Honorare für DMC(Data Monitoring Committee)Tätigkeit und/oder Forschungsunterstützung von folgenden Unternehmen: Aegerion, Akcea, Amarin, Amgen, Berlin-Chemie, Boehringer-Ingelheim, Ionis, MSD, Omniamed, Pfizer, Regeneron, Sanofi.

\section{Literatur}

1. Bhatt DL et al (2018) Cardiovascular risk reduction with icosapent ethyl for hypertriglyceridemia. N Engl J Med https://doi.org/10.1056/ NEJMoa1812792

2. Rawshani A et al (2017) Mortality and cardiovascular disease in type 1 and type 2 diabetes. N Engl J Med 376:1407-1418 